\title{
Eisenmenger Syndrome Complicated by Pulmonary Artery Dissection
}

\author{
Jun Meng ${ }^{1}$ Yongjun Qian ${ }^{1}$ Xijun Xiao $^{1}$ \\ ${ }^{1}$ Department of Cardiovascular Surgery, West China Hospital, Sichuan \\ University, Chengdu, Province of China \\ Thorac Cardiovasc Surg 2012;60(S2):e1-e2.
}

Address for correspondence Dr. Xijun Xiao, M.D., Ph.D., Department of Cardiovascular Surgery, West China Hospital, Sichuan University, No.37, Guoxue Alley, Chengdu, Sichuan Province, Chengdu 610041, Province of China (e-mail: xiaoxijun547@163.com).

\author{
Abstract \\ Keywords \\ - cardiac \\ - congenital heart \\ disease \\ - CHD \\ - echocardiography (all \\ modalities, \\ applications)
}

This report describes a 40-year-old male patient diagnosed with pulmonary artery dissection involving both the left and right branches. The patient also had Eisenmenger syndrome secondary to an uncorrected patent ductus arteriosus. Diagnosis was facilitated through the use of transthoracic echocardiography and computed tomography. Due to the lack of definite guidelines for the optimal treatment strategy of this condition together with the poor condition of the patient, medical management was elected. Not surprisingly, the patient died suddenly only 7 days after the presentation.
A 40-year-old male patient with Eisenmenger syndrome secondary to an uncorrected patent ductus arteriosus (PDA) was admitted to the emergency room due to chest pain and dyspnea. On admission, oxygen saturation was $75 \%$ and blood chemistry analysis results were normal. An electrocardiogram showed left ventricular hypertrophy and thoracic radiographs confirmed the presence of cardiomegaly with pulmonary congestion. Transthoracic echocardiography (TTE) confirmed the presence of a large PDA with a right to

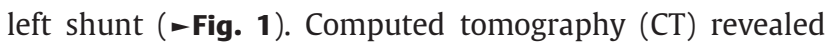
dilatation of the main pulmonary artery and a dissection involving both the left and right pulmonary branches (-Fig. 2). The patient was treated conservatively and died suddenly 7 days after the presentation.

\section{Discussion}

Pulmonary artery dissection is a rare but life-threatening event in which the cause and pathogenesis are unclear. Pulmonary artery dissection is commonly associated with congenital heart disease accompanied by pulmonary hypertension. A PDA is the most frequent congenital heart disease associated with pulmonary artery dissection. ${ }^{1-4}$ It is reasonable to hypothesize that the high-flow rates in combination with secondary pulmonary hypertension associated with

received

May 21, 2011

accepted after revision

June 14, 2011

published online

January 3, 2012

a PDA predispose the pulmonary arteries to cystic medial necrosis. Histologic findings in patients with pulmonary hypertension have identified the presence of cystic medial necrosis in a significant number of cases, which is characterized by the extensive fragmentation of the elastin and collagen skeleton of blood vessels with pooling of mucopolysaccharides. ${ }^{5}$

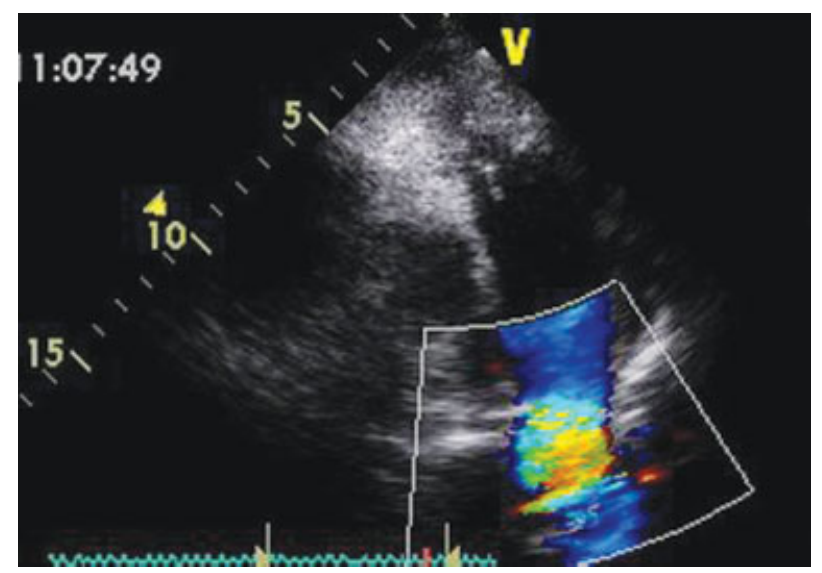

Figure 1 TTE showing a large patent ductus arteriosus with right to left shunt.

(c) 2012 Georg Thieme Verlag KG Stuttgart · New York
DOI http://dx.doi.org/ 10.1055/s-0031-1293606. ISSN 2194-7635. 


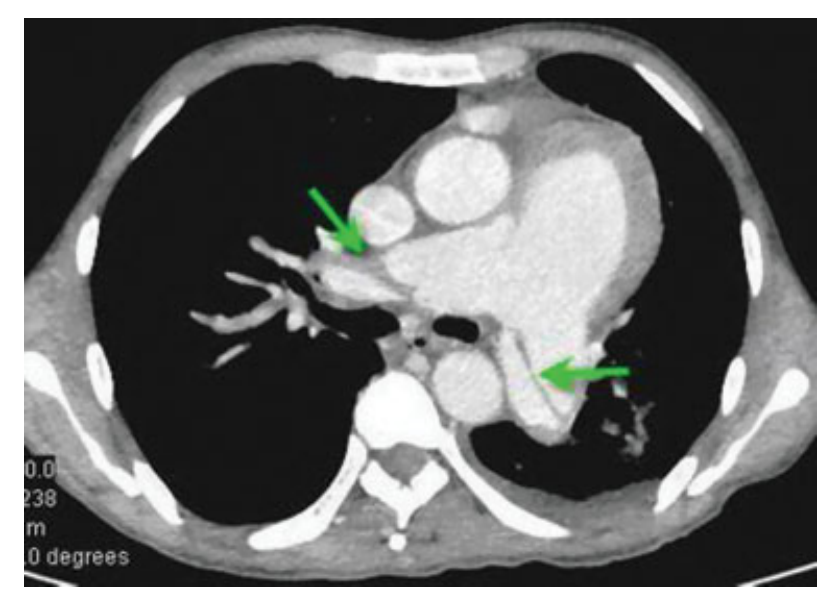

Figure 2 CT showing dilatation of the main pulmonary artery and the dissection involved left and right pulmonary artery (arrow).

The symptoms of pulmonary artery dissection are nonspecific. The most common symptoms are chest pain, dyspnea, cyanosis, hemorrhagic shock, acute pericardial tamponade, and sudden death. In the patient described herein, the clinical diagnosis was facilitated because of the
TTE and CT results. Nonetheless, the clinical decision regarding the treatment was difficult. Surgical repair seemed almost mandatory because of the exceedingly high risk of rupture of the dissecting, high-pressure pulmonary artery aneurysms; however, the chances of the patient surviving such an operation were considered very poor due to the Eisenmenger syndrome.

\section{References}

1 Inayama Y, Nakatani Y, Kitamura H. Pulmonary artery dissection in patients without underlying pulmonary hypertension. Histopathology 2001;38(5):435-442

2 Deterling RA Jr, Clagett OT. Aneurysm of the pulmonary artery; review of the literature and report of a case. Am Heart J 1947;34 (4):471-499

3 Khattar RS, Fox DJ, Alty JE, Arora A. Pulmonary artery dissection: an emerging cardiovascular complication in surviving patients with chronic pulmonary hypertension. Heart 2005;91(2):142145

4 Graham JK, Shehata B. Sudden death due to dissecting pulmonary artery aneurysm: a case report and review of the literature. Am J Forensic Med Pathol 2007;28(4):342-344

5 Sardesai SH, Marshall RJ, Farrow RJ, Mourant AJ. Dissecting aneurysm of the pulmonary artery in a case of unoperated patent ductus arteriosus. Eur Heart J 1990;11(7):670-673 